\title{
CDISC Special Purpose Class
}

National Cancer Institute

\section{Source}

National Cancer Institute. CDISC Special Purpose Class. NCI Thesaurus. Code C103377.

This SDT M class contains a set of domains which do not conform to the Findings, Events or Interventions observation classes. The domains included are DM, CO, SE and SV. 